\title{
A NEW WEBER-TYPE TRANSFORM
}

\author{
BY \\ R. K. MICHAEL THAMBYNAYAGAM AND TAREK M. HABASHY \\ Schlumberger-Doll Research, Old Quarry Road, Ridgefield, CT 06877-4108
}

\begin{abstract}
In this paper we introduce a new Weber-type transform pair for the representation of a function $f(r)$ defined over the domain $a \leq r<\infty$ and which satisfies the Robin mixed boundary condition $f(a)+\lambda f^{\prime}(a)=0$. The orthogonality relationships of the transform kernels are derived in both the spatial and the spectral domains as well as Parseval's theorem. We apply this new Weber-type transform pair to solve a mixed boundary value problem in a system of planar layers.
\end{abstract}

1. Introduction. In many practical applications, integral transform methods have been a powerful analytical tool in solving mixed boundary value problems. The most important advantage of such transform methods is that they provide a means of reducing the degree of complexity of solving a problem allowing, in some cases, analytical or semianalytical solutions. Also in hybrid numerical techniques, integral transforms can permit the reduction of the dimensionality of the problem at hand, hence significantly reducing the computation cost.

In the next section and using the notations of this paper, we review some known material on Weber transform pairs [1]-[3] for the representation of a function $f(r)$ defined over the domain $a \leq r<\infty$ and which satisfies either a Dirichlet boundary condition $f(a)=0$ or a Neumann boundary condition $f^{\prime}(a)=0$. Weber's integral theorem and its proof for circular cylinder functions of unrestricted order with Dirichlet and Neumann boundary conditions have been established by Watson [2]. In the third and fourth sections we introduce a new Weber-type transform pair for the representation of a function $f(r)$ defined over the domain $a \leq r<\infty$ and which satisfies the Robin mixed boundary condition $f(a)+\lambda f^{\prime}(a)=0$. In the last section, we apply this new Weber-type transform pair to solve a mixed boundary value problem in a system of planar layers.

Received May, 2001.

2000 Mathematics Subject Classification. Primary 65R10, 86-08.

Key words and phrases. Integral transforms, Weber transforms, mixed boundary value problems, Green functions, Hankel transforms.

E-mail address: thamby@abingdon.geoquest.slb.com

E-mail address: thabashy@ridgefield.oilfield.slb.com 
2. The Dirichlet-Weber and Neumann-Weber transforms. For a function $f(r)$ defined over the domain $a \leq r<\infty$ and subject to the condition that:

$$
\int_{a}^{\infty} d r \sqrt{r} f(r)
$$

exists and is absolutely convergent, its Weber transform [1]-[3] is given by the following pair:

$$
\begin{aligned}
\tilde{f}_{\nu}(\xi) & =\int_{a}^{\infty} d r r C_{\nu}(r, \xi) f(r) \\
f(r) & =\int_{0}^{\infty} d \xi \xi C_{\nu}(r, \xi) \tilde{f}_{\nu}(\xi)
\end{aligned}
$$

where $\nu \geq-1 / 2$ and $C_{\nu}(r, \xi)$ is the kernel of the Weber transform given by:

$$
C_{\nu}(r, \xi)=\frac{1}{N_{\nu}(\xi a)}\left[Y_{\nu}(\xi a) J_{\nu}(\xi r)-J_{\nu}(\xi a) Y_{\nu}(\xi r)\right]
$$

for a function $f(r)$ that satisfies the Dirichlet boundary condition $f(a)=0$, in which case we refer to the Weber transform as the Dirichlet-Weber Transform (DWT). On the other hand, for a function $f(r)$ that satisfies the Neumann boundary condition $f^{\prime}(a)=0$, the kernel $C_{\nu}(r, \xi)$ is given by:

$$
C_{\nu}(r, \xi)=\frac{1}{M_{\nu}(\xi a)}\left[Y_{\nu}^{\prime}(\xi a) J_{\nu}(\xi r)-J_{\nu}^{\prime}(\xi a) Y_{\nu}(\xi r)\right]
$$

in which case we refer to the Weber transform as the Neumann-Weber Transform (NWT). The normalization coefficients, $N_{\nu}(\xi a)$ and $M_{\nu}(\xi a)$, are given by:

$$
\begin{aligned}
& N_{\nu}(\xi a)=\left[J_{\nu}^{2}(\xi a)+Y_{\nu}^{2}(\xi a)\right]^{1 / 2} \\
& M_{\nu}(\xi a)=\left[J_{\nu}^{\prime 2}(\xi a)+Y_{\nu}^{\prime 2}(\xi a)\right]^{1 / 2} .
\end{aligned}
$$

This kernel satisfies the following partial differential equation:

$$
\left[\frac{\partial^{2}}{\partial r^{2}}+\frac{1}{r} \frac{\partial}{\partial r}+\left(\xi^{2}-\frac{\nu^{2}}{r^{2}}\right)\right] C_{\nu}(r, \xi)=0
$$

subject to the boundary condition:

$$
C_{\nu}(a, \xi)=0, \quad \text { for the DWT }
$$

and

$$
C_{\nu}^{\prime}(a, \xi)=0, \quad \text { for the NWT. }
$$

The prime indicates differentiation with respect to the argument (or first argument). The Weber transform kernel, $C_{\nu}(r, \xi)$, also satisfies the following orthogonality relationships:

$$
\begin{aligned}
\int_{a}^{\infty} d r r C_{\nu}\left(r, \xi_{1}\right) C_{\nu}\left(r, \xi_{2}\right) & =\frac{1}{\sqrt{\xi_{1} \xi_{2}}} \delta\left(\xi_{1}-\xi_{2}\right) \\
\int_{0}^{\infty} d \xi \xi C_{\nu}\left(r_{1}, \xi\right) C_{\nu}\left(r_{2}, \xi\right) & =\frac{1}{\sqrt{r_{1} r_{2}}} \delta\left(r_{1}-r_{2}\right) .
\end{aligned}
$$

Parseval's theorem states that:

$$
\int_{a}^{\infty} d r r f(r) g(r)=\int_{0}^{\infty} d \xi \xi \tilde{f}_{\nu}(\xi) \tilde{g}_{\nu}(\xi)
$$


where $\tilde{f}_{\nu}(\xi)$ and $\tilde{g}_{\nu}(\xi)$ are the Weber transforms of the two functions $f(r)$ and $g(r)$, respectively. It is implied that $f(r)$ and $g(r)$ both satisfy either the Dirichlet or the Neumann boundary conditions and that $\tilde{f}_{\nu}(\xi)$ and $\tilde{g}_{\nu}(\xi)$ are their corresponding DWTs or NWTs, respectively.

Defining the operator $\mathcal{L}_{\nu}\{f(r)\}$ by:

$$
\mathcal{L}_{\nu}\{f(r)\}=\frac{\partial^{2} f(r)}{\partial r^{2}}+\frac{1}{r} \frac{\partial f(r)}{\partial r}-\frac{\nu^{2}}{r^{2}} f(r),
$$

the principal operational property of the Weber transform is given by:

$$
\begin{aligned}
\int_{a}^{\infty} d r r \mathcal{L}_{\nu}\{f(r)\} C_{\nu}(r, \xi) & =a f^{\prime}(a) C_{\nu}(a, \xi)-a f(a) C_{\nu}^{\prime}(a, \xi)-\xi^{2} \tilde{f}_{\nu}(\xi) \\
& =-\xi^{2} \tilde{f}_{\nu}(\xi),
\end{aligned}
$$

where we have made use of the conditions $f(a)=0$ and $C_{\nu}(a, \xi)=0$ for the DirichletWeber transform and of the conditions $f^{\prime}(a)=0$ and $C_{\nu}^{\prime}(a, \xi)=0$ for the NeumannWeber transform.

In the limit when $a \rightarrow 0$, we obtain $C_{\nu}(r, \xi) \rightarrow J_{\nu}(\xi r)$ and the Weber transform reduces to the Hankel transform [4].

3. A new Weber-type transform. In this section we develop a new Weber-type transform for the representation of a function $f(r)$ defined over the domain $a \leq r<\infty$ and which satisfies Robin mixed boundary condition $f(a)+\lambda f^{\prime}(a)=0$. The function $f(r)$ is subject to the condition that:

$$
\int_{a}^{\infty} d r \sqrt{r} f(r)
$$

exists and is absolutely convergent. The new Weber-type transform for the function $f(r)$ is given by the following pair:

$$
\begin{aligned}
\tilde{f}_{\nu}(\xi) & =\int_{a}^{\infty} d r r \mathcal{D}_{\nu}(r, \xi) f(r) \\
f(r) & =\int_{0}^{\infty} d \xi \xi \mathcal{D}_{\nu}(r, \xi) \tilde{f}_{\nu}(\xi),
\end{aligned}
$$

where $\nu \geq-1 / 2$ and $\mathcal{D}_{\nu}(r, \xi)$ is the kernel of the transform given by:

$$
\mathcal{D}_{\nu}(r, \xi)=\frac{1}{P_{\nu}(\xi)}\left[\left\{Y_{\nu}(\xi a)+\lambda \xi Y_{\nu}^{\prime}(\xi a)\right\} J_{\nu}(\xi r)-\left\{J_{\nu}(\xi a)+\lambda \xi J_{\nu}^{\prime}(\xi a)\right\} Y_{\nu}(\xi r)\right]
$$

with

$$
P_{\nu}(\xi)=\left[\left\{J_{\nu}(\xi a)+\lambda \xi J_{\nu}^{\prime}(\xi a)\right\}^{2}+\left\{Y_{\nu}(\xi a)+\lambda \xi Y_{\nu}^{\prime}(\xi a)\right\}^{2}\right]^{1 / 2} .
$$

This kernel satisfies the following equation:

$$
\left[\frac{\partial^{2}}{\partial r^{2}}+\frac{1}{r} \frac{\partial}{\partial r}+\left(\xi^{2}-\frac{\nu^{2}}{r^{2}}\right)\right] \mathcal{D}_{\nu}(r, \xi)=0
$$

with

$$
\mathcal{D}_{\nu}(a, \xi)+\lambda \mathcal{D}_{\nu}^{\prime}(a, \xi)=0
$$


It also satisfies the following orthogonality relationships:

$$
\begin{aligned}
\int_{a}^{\infty} d r r \mathcal{D}_{\nu}\left(r, \xi_{1}\right) \mathcal{D}_{\nu}\left(r, \xi_{2}\right) & =\frac{1}{\sqrt{\xi_{1} \xi_{2}}} \delta\left(\xi_{1}-\xi_{2}\right) \\
\int_{0}^{\infty} d \xi \xi \mathcal{D}_{\nu}\left(r_{1}, \xi\right) \mathcal{D}_{\nu}\left(r_{2}, \xi\right) & =\frac{1}{\sqrt{r_{1} r_{2}}} \delta\left(r_{1}-r_{2}\right)
\end{aligned}
$$

Parseval's theorem states that:

$$
\int_{a}^{\infty} d r r f(r) g(r)=\int_{0}^{\infty} d \xi \xi \tilde{f}_{\nu}(\xi) \tilde{g}_{\nu}(\xi),
$$

where $\tilde{f}_{\nu}(\xi)$ and $\tilde{g}_{\nu}(\xi)$ are the new Weber-type transforms of the two functions $f(r)$ and $g(r)$, respectively, which satisfy Robin mixed boundary conditions $f(a)+\lambda f^{\prime}(a)=0$ and $g(a)+\lambda g^{\prime}(a)=0$, respectively.

The principal operational property of this Weber-type transform is:

$$
\begin{aligned}
\int_{a}^{\infty} d r r \mathcal{L}_{\nu}\{f(r)\} \mathcal{D}_{\nu}(r, \xi) & =a\left[f^{\prime}(a) \mathcal{D}_{\nu}(a, \xi)-f(a) \mathcal{D}_{\nu}^{\prime}(a, \xi)\right]-\xi^{2} \tilde{f}_{\nu}(\xi) \\
& =-a \mathcal{D}_{\nu}^{\prime}(a, \xi)\left[f(a)+\lambda f^{\prime}(a)\right]-\xi^{2} \tilde{f}_{\nu}(\xi) \\
& =-\xi^{2} \tilde{f}_{\nu}(\xi)
\end{aligned}
$$

where we have used the conditions $\mathcal{D}_{\nu}(a, \xi)+\lambda \mathcal{D}_{\nu}^{\prime}(a, \xi)=0$ and $f(a)+\lambda f^{\prime}(a)=0$. In the limit when $\lambda \rightarrow 0$, we obtain $\mathcal{D}_{\nu}(r, \xi) \rightarrow C_{\nu}(r, \xi)$ and the new Weber-type transform reduces to the Dirichlet-Weber transform. Similarly, in the limit when $\lambda \rightarrow \infty$, we have $\mathcal{D}_{\nu}(r, \xi) \rightarrow C_{\nu}(r, \xi)$ and the new Weber-type transform reduces to the Neumann-Weber transform. Finally, in the limit when $a \rightarrow 0$, we obtain $\mathcal{D}_{\nu}(r, \xi) \rightarrow J_{\nu}(\xi r)$ and the new Weber-type transform reduces to the Hankel transform.

4. Proof of the orthogonality relationships. $\mathcal{D}_{\nu}\left(r, \xi_{1}\right)$ and $\mathcal{D}_{\nu}\left(r, \xi_{2}\right)$ satisfy the following two equations:

$$
\begin{aligned}
& {\left[\frac{1}{r} \frac{\partial}{\partial r}\left(r \frac{\partial}{\partial r}\right)+\left(\xi_{1}^{2}-\frac{\nu^{2}}{r^{2}}\right)\right] \mathcal{D}_{\nu}\left(r, \xi_{1}\right)=0} \\
& {\left[\frac{1}{r} \frac{\partial}{\partial r}\left(r \frac{\partial}{\partial r}\right)+\left(\xi_{2}^{2}-\frac{\nu^{2}}{r^{2}}\right)\right] \mathcal{D}_{\nu}\left(r, \xi_{2}\right)=0 .}
\end{aligned}
$$

Multiplying Eq. (27) by $\mathcal{D}_{\nu}\left(r, \xi_{2}\right)$ and Eq. $(28)$ by $\mathcal{D}_{\nu}\left(r, \xi_{1}\right)$ and subtracting, we obtain:

$$
\left(\xi_{2}^{2}-\xi_{1}^{2}\right) \mathcal{D}_{\nu}\left(r, \xi_{1}\right) \mathcal{D}_{\nu}\left(r, \xi_{2}\right)=\frac{1}{r} \frac{\partial}{\partial r}\left[r\left\{\mathcal{D}_{\nu}\left(r, \xi_{2}\right) \mathcal{D}_{\nu}^{\prime}\left(r, \xi_{1}\right)-\mathcal{D}_{\nu}\left(r, \xi_{1}\right) \mathcal{D}_{\nu}^{\prime}\left(r, \xi_{2}\right)\right\}\right] .
$$

Multiplying by $r$ and integrating from $a$ to $b$ (later set to $\infty$ in the limit), we get:

$$
\begin{aligned}
\left(\xi_{2}^{2}-\xi_{1}^{2}\right) \int_{a}^{b} d r r \mathcal{D}_{\nu}\left(r, \xi_{1}\right) \mathcal{D}_{\nu}\left(r, \xi_{2}\right)= & {\left[r\left\{\mathcal{D}_{\nu}\left(r, \xi_{2}\right) \mathcal{D}_{\nu}^{\prime}\left(r, \xi_{1}\right)-\mathcal{D}_{\nu}\left(r, \xi_{1}\right) \mathcal{D}_{\nu}^{\prime}\left(r, \xi_{2}\right)\right\}\right]_{a}^{b} } \\
= & b\left[\mathcal{D}_{\nu}\left(b, \xi_{2}\right) \mathcal{D}_{\nu}^{\prime}\left(b, \xi_{1}\right)-\mathcal{D}_{\nu}\left(b, \xi_{1}\right) \mathcal{D}_{\nu}^{\prime}\left(b, \xi_{2}\right)\right] \\
& -a\left[\mathcal{D}_{\nu}\left(a, \xi_{2}\right) \mathcal{D}_{\nu}^{\prime}\left(a, \xi_{1}\right)-\mathcal{D}_{\nu}\left(a, \xi_{1}\right) \mathcal{D}_{\nu}^{\prime}\left(a, \xi_{2}\right)\right] \\
= & b\left[\mathcal{D}_{\nu}\left(b, \xi_{2}\right) \mathcal{D}_{\nu}^{\prime}\left(b, \xi_{1}\right)-\mathcal{D}_{\nu}\left(b, \xi_{1}\right) \mathcal{D}_{\nu}^{\prime}\left(b, \xi_{2}\right)\right]
\end{aligned}
$$


where we have used:

$$
\begin{aligned}
& \mathcal{D}_{\nu}(a, \xi)=\frac{2 \lambda}{\pi a P_{\nu}(\xi)} \\
& \mathcal{D}_{\nu}^{\prime}(a, \xi)=-\frac{2}{\pi a P_{\nu}(\xi)} .
\end{aligned}
$$

In the limit of large $b(b \rightarrow \infty)$, we have [5, p. 364]:

$$
\begin{aligned}
& J_{\nu}(\xi b) \rightarrow \sqrt{\frac{2}{\pi \xi b}} \cos \left\{\xi b-\left(\nu+\frac{1}{2}\right) \frac{\pi}{2}\right\} \\
& J_{\nu}^{\prime}(\xi b) \rightarrow-\sqrt{\frac{2}{\pi \xi b}} \sin \left\{\xi b-\left(\nu+\frac{1}{2}\right) \frac{\pi}{2}\right\} \\
& Y_{\nu}(\xi b) \rightarrow \sqrt{\frac{2}{\pi \xi b}} \sin \left\{\xi b-\left(\nu+\frac{1}{2}\right) \frac{\pi}{2}\right\} \\
& Y_{\nu}^{\prime}(\xi b) \rightarrow \sqrt{\frac{2}{\pi \xi b}} \cos \left\{\xi b-\left(\nu+\frac{1}{2}\right) \frac{\pi}{2}\right\} .
\end{aligned}
$$

Hence,

$$
\begin{aligned}
& \int_{a}^{b} d r r \mathcal{D}_{\nu}\left(r, \xi_{1}\right) \mathcal{D}_{\nu}\left(r, \xi_{2}\right) \rightarrow \frac{1}{P_{\nu}\left(\xi_{1}\right) P_{\nu}\left(\xi_{2}\right)} \frac{1}{\pi \sqrt{\xi_{1} \xi_{2}}} \\
& \times\left[\left\{A_{\nu}\left(\xi_{1}\right) A_{\nu}\left(\xi_{2}\right)+B_{\nu}\left(\xi_{1}\right) B_{\nu}\left(\xi_{2}\right)\right\} \frac{\sin \left\{\left(\xi_{2}-\xi_{1}\right) b\right\}}{\xi_{2}-\xi_{1}}\right. \\
&+\left\{A_{\nu}\left(\xi_{1}\right) A_{\nu}\left(\xi_{2}\right)-B_{\nu}\left(\xi_{1}\right) B_{\nu}\left(\xi_{2}\right)\right\} \frac{\sin \left\{\left(\xi_{2}+\xi_{1}\right) b\right\}}{\xi_{2}+\xi_{1}} \\
&+\left\{A_{\nu}\left(\xi_{1}\right) B_{\nu}\left(\xi_{2}\right)-B_{\nu}\left(\xi_{1}\right) A_{\nu}\left(\xi_{2}\right)\right\} \frac{\cos \left\{\left(\xi_{2}-\xi_{1}\right) b\right\}}{\xi_{2}-\xi_{1}} \\
&\left.+\left\{A_{\nu}\left(\xi_{1}\right) B_{\nu}\left(\xi_{2}\right)+B_{\nu}\left(\xi_{1}\right) A_{\nu}\left(\xi_{2}\right)\right\} \frac{\cos \left\{\left(\xi_{2}+\xi_{1}\right) b\right\}}{\xi_{2}+\xi_{1}}\right]
\end{aligned}
$$

where

$$
\begin{aligned}
& A_{\nu}(\xi)=Y_{\nu}(\xi a)+\lambda \xi Y_{\nu}^{\prime}(\xi a) \\
& B_{\nu}(\xi)=J_{\nu}(\xi a)+\lambda \xi J_{\nu}^{\prime}(\xi a)
\end{aligned}
$$

From the integral

$$
\int_{0}^{\infty} d r e^{i \xi r}=\pi \delta(\xi)+\frac{i}{\xi}
$$

we obtain:

$$
\pi \delta(\xi)=\int_{0}^{\infty} d r \cos (\xi r)=\lim _{b \rightarrow \infty} \int_{0}^{b} d r \cos (\xi r)=\lim _{b \rightarrow \infty} \frac{\sin \{\xi b\}}{\xi}
$$

and

$$
\frac{1}{\xi}=\int_{0}^{\infty} d r \sin (\xi r)=\lim _{b \rightarrow \infty} \int_{0}^{b} d r \sin (\xi r)=\frac{1}{\xi}-\lim _{b \rightarrow \infty} \frac{\cos \{\xi b\}}{\xi} \Rightarrow \lim _{b \rightarrow \infty} \frac{\cos \{\xi b\}}{\xi}=0
$$


Taking the limit of Eq. (37) as $b \rightarrow \infty$, we finally obtain the first orthogonality relationship of Eq. (23):

$$
\int_{a}^{\infty} d r r \mathcal{D}_{\nu}\left(r, \xi_{1}\right) \mathcal{D}_{\nu}\left(r, \xi_{2}\right)=\frac{1}{\sqrt{\xi_{1} \xi_{2}}} \delta\left(\xi_{1}-\xi_{2}\right)
$$

where we have used

$$
P_{\nu}^{2}(\xi)=A_{\nu}^{2}(\xi)+B_{\nu}^{2}(\xi)
$$

The proof of the second orthogonality relationship of Eq. (24) follows directly from the first orthogonality relationship of Eq. (23). From the first orthogonality relationship, we have shown that:

$$
\tilde{f}_{\nu}(\xi)=\int_{a}^{\infty} d r r \mathcal{D}_{\nu}(r, \xi) \int_{0}^{\infty} d \xi^{\prime} \xi^{\prime} \mathcal{D}_{\nu}\left(r, \xi^{\prime}\right) \tilde{f}_{\nu}\left(\xi^{\prime}\right) .
$$

Defining

$$
f(r)=\int_{0}^{\infty} d \xi^{\prime} \xi^{\prime} \mathcal{D}_{\nu}\left(r, \xi^{\prime}\right) \tilde{f}_{\nu}\left(\xi^{\prime}\right)
$$

we obtain from Eq. (45):

$$
\tilde{f}_{\nu}(\xi)=\int_{a}^{\infty} d r r \mathcal{D}_{\nu}(r, \xi) f(r)
$$

and substituting back in Eq. (46), we obtain:

$$
f(r)=\int_{0}^{\infty} d \xi \xi \mathcal{D}_{\nu}(r, \xi) \int_{a}^{\infty} d r^{\prime} r^{\prime} \mathcal{D}_{\nu}\left(r^{\prime}, \xi\right) f\left(r^{\prime}\right) .
$$

Upon rearrangement, we have:

$$
f(r)=\int_{a}^{\infty} d r^{\prime} r^{\prime} f\left(r^{\prime}\right) \int_{0}^{\infty} d \xi \xi \mathcal{D}_{\nu}(r, \xi) \mathcal{D}_{\nu}\left(r^{\prime}, \xi\right)
$$

which implies

$$
\int_{0}^{\infty} d \xi \xi \mathcal{D}_{\nu}(r, \xi) \mathcal{D}_{\nu}\left(r^{\prime}, \xi\right)=\frac{1}{r^{\prime}} \delta\left(r^{\prime}-r\right)=\frac{1}{\sqrt{r r^{\prime}}} \delta\left(r^{\prime}-r\right),
$$

resulting in the second orthogonality relationship of Eq. (24).

5. Green function in an unbounded medium. As an application of the use of the new Weber-type transform to the solution of partial differential equations, we develop an expression for the Green function, $g\left(\overline{\mathbf{R}}, \overline{\mathbf{R}}^{\prime}\right)$, in an infinite unbounded medium. The Green function satisfies the following partial differential equation:

$$
\left(\nabla^{2}-\gamma^{2}\right) g\left(\overline{\mathbf{R}}, \overline{\mathbf{R}}^{\prime}\right)=-\delta\left(\overline{\mathbf{R}}-\overline{\mathbf{R}}^{\prime}\right), \quad r, r^{\prime} \geq a
$$

with the boundary condition

$$
\left[g\left(\overline{\mathbf{R}}, \overline{\mathbf{R}}^{\prime}\right)+\lambda \frac{\partial}{\partial r} g\left(\overline{\mathbf{R}}, \overline{\mathbf{R}}^{\prime}\right)\right]_{r=a}=0
$$

where $\gamma$ is a wavenumber and $\lambda$ is a constant. Expanding the Green function as a Fourier series along the $\phi$-direction and in terms of the new Weber-type transform along the $r$-direction, we obtain:

$$
g\left(\overline{\mathbf{R}}, \overline{\mathbf{R}}^{\prime}\right)=\sum_{\nu} e^{i \nu\left(\phi-\phi^{\prime}\right)} \int_{0}^{\infty} d \xi \xi \mathcal{D}_{\nu}(r, \xi) \tilde{g}_{\nu}\left(\xi, z ; \overline{\mathbf{R}}^{\prime}\right) .
$$


Substituting from Eq. (53) into the PDE of Eq. (51) and using the properties of the new Weber-type transform pairs derived in the previous sections, one can easily show that $\tilde{g}_{\nu}\left(\xi, z ; \overline{\mathbf{R}}^{\prime}\right)$ satisfies the following ODE:

$$
\left(\frac{d^{2}}{d z^{2}}-\alpha^{2}\right) \tilde{g}_{\nu}\left(\xi, z ; \overline{\mathbf{R}}^{\prime}\right)=-\frac{1}{4 \pi} \mathcal{D}_{\nu}\left(r^{\prime}, \xi\right) \delta\left(z-z^{\prime}\right),
$$

where

$$
\alpha^{2}=\xi^{2}+\gamma^{2}
$$

Equation (54) has the solution

$$
\tilde{g}_{\nu}\left(\xi, z ; \overline{\mathbf{R}}^{\prime}\right)=\frac{1}{4 \pi \alpha} \mathcal{D}_{\nu}\left(r^{\prime}, \xi\right) e^{-\alpha\left|z-z^{\prime}\right|}
$$

and the Green function is therefore given by

$$
g\left(\overline{\mathbf{R}}, \overline{\mathbf{R}}^{\prime}\right)=\frac{1}{4 \pi} \sum_{\nu} e^{i \nu\left(\phi-\phi^{\prime}\right)} \int_{0}^{\infty} d \xi \frac{\xi}{\alpha} \mathcal{D}_{\nu}(r, \xi) \mathcal{D}_{\nu}\left(r^{\prime}, \xi\right) e^{-\alpha\left|z-z^{\prime}\right|} .
$$

6. Green function in a layered system. In this section we develop an expression for Green function in a system of planar layers in the presence of the cylindrical surface, $r=a$, where the Green function satisfies Robin mixed boundary condition given by Eq. (52). The source region is located in the layer $-h_{0}^{d} \leq z, z^{\prime} \leq h_{0}^{u}$ whose thickness is $H=h_{0}^{d}+h_{0}^{u}$. There are $N^{u}$ layers above the source region and $N^{d}$ layers below. The thickness of the $n$-th upper layer is $h_{n}^{u}$ and that of the $n$-th lower layer is $h_{n}^{d}$ (see Fig. 1).

We assume that the Green function satisfies the following set of generic boundary conditions at a boundary located at $z=z_{b}$ separating the two layers above and below:

$$
\begin{aligned}
{\left[g\left(\overline{\mathbf{R}}, \overline{\mathbf{R}}^{\prime}\right)\right]_{z=z_{b}-} } & =\left[g\left(\overline{\mathbf{R}}, \overline{\mathbf{R}}^{\prime}\right)\right]_{z=z_{b}+} \\
{\left[\eta \frac{\partial}{\partial r} g\left(\overline{\mathbf{R}}, \overline{\mathbf{R}}^{\prime}\right)\right]_{z=z_{b}-} } & =\left[\eta \frac{\partial}{\partial r} g\left(\overline{\mathbf{R}}, \overline{\mathbf{R}}^{\prime}\right)\right]_{z=z_{b}+},
\end{aligned}
$$

where $\eta$ is the material property of the medium which encounters a change across the boundary at $z=z_{b}$ separating the two layers.

Following the approach in [6], one can show that for observation points in the source layer, $-h_{0}^{d} \leq z, z^{\prime} \leq h_{0}^{u}$, the Green function is given by:

$$
\begin{aligned}
& g\left(\overline{\mathbf{R}}, \overline{\mathbf{R}}^{\prime}\right)= \frac{1}{4 \pi} \sum_{\nu} e^{i \nu\left(\phi-\phi^{\prime}\right)} \int_{0}^{\infty} d \xi \frac{\xi}{\alpha_{0}} \mathcal{D}_{\nu}(r, \xi) \mathcal{D}_{\nu}\left(r^{\prime}, \xi\right)\left[e^{-\alpha_{0}\left|z-z^{\prime}\right|}\right. \\
&+\frac{1}{1-R_{0}^{u} R_{0}^{d} e^{-2 \alpha_{0} H}}\left\{\left(1+R_{0}^{u} e^{-2 \alpha_{0} h_{0}^{u}}\right) R_{0}^{d} e^{-\alpha_{0}\left(2 h_{0}^{d}+z\right)}\right. \\
&\left.\left.+\left(1+R_{0}^{d} e^{-2 \alpha_{0} h_{0}^{d}}\right) R_{0}^{u} e^{-\alpha_{0}\left(2 h_{0}^{u}-z\right)}\right\}\right]
\end{aligned}
$$

where $R_{0}^{u}$ and $R_{0}^{d}$ are computed using the following recurrence relationships [6]:

$$
R_{n}^{u, d}=\frac{r_{n, n+1}^{u, d}+R_{n+1}^{u, d} e^{-2 \alpha_{n+1}^{u, d} h_{n+1}^{u, d}}}{1+r_{n, n+1}^{u, d} R_{n+1}^{u, d} e^{-2 \alpha_{n+1}^{u, d} h_{n+1}^{u, d}}}, \quad n=N^{u, d}-1, N^{u, d}-2, \ldots, 1,0
$$




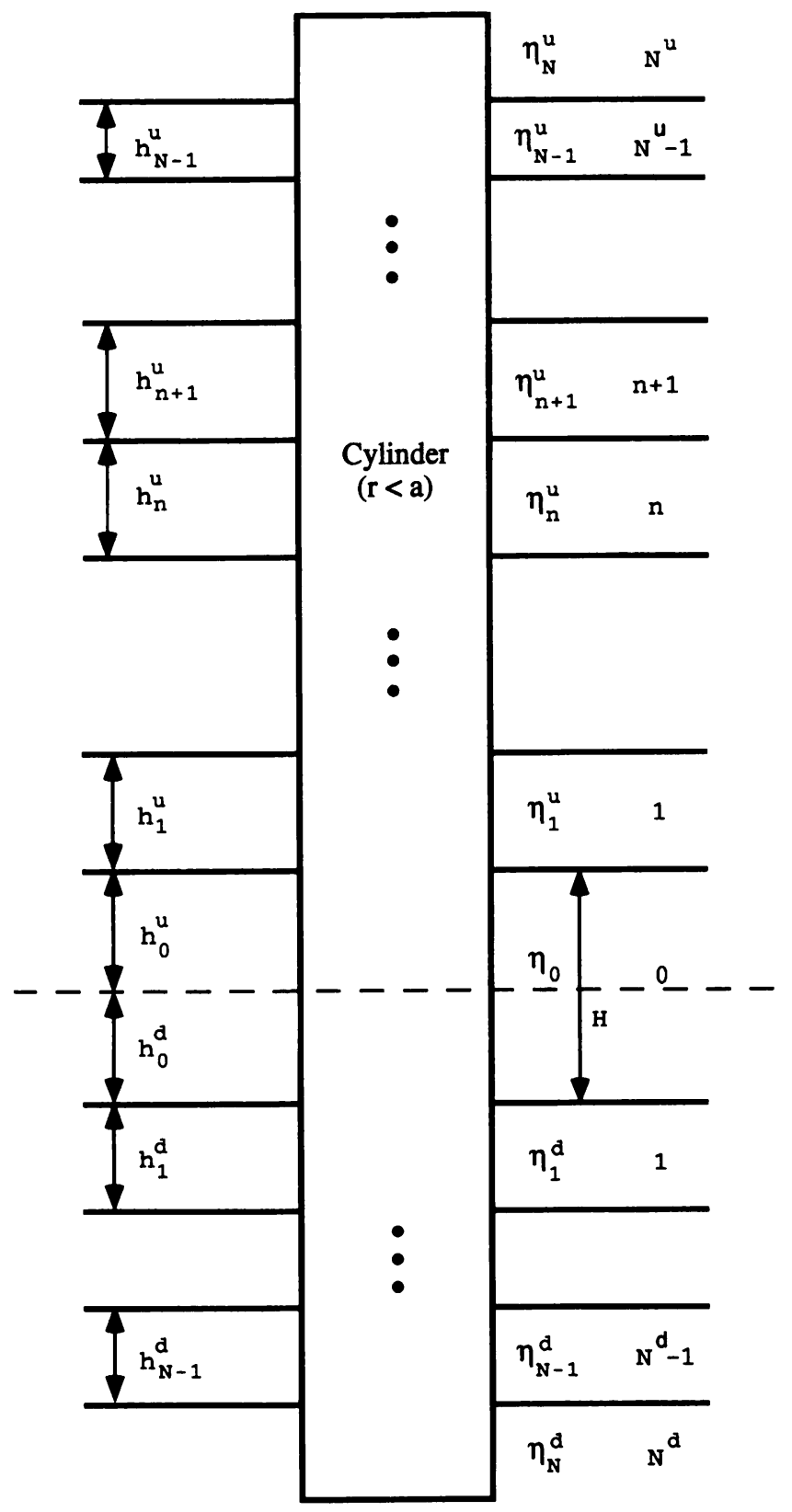

FIG. 1. Schematic of a system of planar layers in the presence of a cylindrical surface.

and

$$
\begin{aligned}
r_{n, n+1}^{u, d} & =\frac{\eta_{n}^{u, d}-\eta_{n+1}^{u, d}}{\eta_{n}^{u, d}+\eta_{n+1}^{u, d}} \\
\alpha_{0}^{2} & =\xi^{2}+\gamma_{0}^{2} \\
{\left[\alpha_{n}^{u, d}\right]^{2} } & =\xi^{2}+\left[\gamma_{n}^{u, d}\right]^{2} .
\end{aligned}
$$


Conclusions. In this paper a new pair of Weber-type transform has been developed which facilitates the solution of mixed boundary value problems for field functions, $f(r)$, defined over the domain $a \leq r<\infty$ and which satisfy Robin mixed boundary condition $f(a)+\lambda f^{\prime}(a)=0$. The properties of the transform kernels have been discussed and their orthogonality relationships have been derived in both the spatial and the spectral domains. Also, the relevant Parseval's theorem was deduced.

Acknowledgments. We thank T. S. Ramakrishnan and the reviewer of this paper for useful suggestions on improving the manuscript.

\section{REFERENCES}

[1] H. Weber, Ueber eine Darstellung willkürliccher Functionen durch Bessel'sche Functionen, Math. Ann. VI, 146 (1873)

[2] G. N. Watson, Theory of Bessel Functions (Cambridge University Press, Cambridge, 1980)

[3] E. C. Titchmarsh, Eigenfunction Expansions Associated with Second-Order Differential Equations (Oxford University Press, Oxford, 1962)

[4] H. Hankel, Die Fourier'schen Reihen und Integrale für Cylinder functionen (original memoirs 1869), Math. Ann. VIII, 467 (1875)

[5] M. Abramowitz and I. A. Stegun, Handbook of Mathematical Functions (Dover Publications, New York, 1970)

[6] F. Kuchuk and T. Habashy, Pressure behavior of laterally composite reservoirs, SPE Journal on Formation Evaluation 12, 47 (1997) 